\title{
FUNDAMENTAL SOLUTION TO THE CAUCHY PROBLEM FOR THE TIME-FRACTIONAL ADVECTION-DIFFUSION EQUATION
}

\author{
Yuriy Povstenko ${ }^{1}$, Joanna Klekot ${ }^{2}$ \\ ${ }^{1}$ Institute of Mathematics and Computer Science, Jan Dtugosz University in Częstochowa \\ Częstochowa, Poland \\ ${ }^{2}$ Institute of Mathematics, Częstochowa University of Technology \\ Czestochowa, Poland \\ ${ }^{1}$ j.povstenko@ajd.czest.pl, ${ }^{2}$ joanna.klekot@im.pcz.pl
}

\begin{abstract}
The one-dimensional time-fractional advection-diffusion equation with the Caputo time derivative is considered. The fundamental solution to the Cauchy problem is obtained using the integral transform technique. The numerical results are illustrated graphically.
\end{abstract}

\section{Introduction}

In the last few decades, a considerable research effort has been expended to the study of fractional differential equations which have many applications in physics, geophysics, geology, chemistry, rheology, engineering and bioengineering (see, for example, [1-8] and references therein). The time-fractional diffusion-wave equation is a mathematical model of important physical phenomena ranging from amorphous, colloid, glassy and porous materials through fractals, random and disordered media to comb structures, dielectrics, semiconductors, polymers and biological systems. The time-fractional advection-diffusion equation can be interpreted in terms of diffusion or heat conduction with additional velocity field, transport processes in porous media and groundwater hydrology. In the case of the fractional advection-diffusion equation, as a rule, different numerical methods have been used to find the solution: the implicit difference method based on the shifted Grünwald approximation [9] and the explicit difference method [10], the fractional variational method [11], the finite volume method [12], etc. In [13, 14] the solution to one-dimensional time-fractional advection-diffusion equation was obtained in terms of the $H$-function.

In this paper, we get the fundamental solution to the Cauchy problem for the time-fractional advection-diffusion equation in terms of the Mittag-Leffler function. The Laplace transform with respect to time and the exponential Fourier transform with respect to the space coordinate are used. The results of numerical calculations are illustrated graphically. 


\section{Formulation of the problem}

Consider the time-fractional advection-diffusion equation

$$
\frac{\partial^{\alpha} T(x, t)}{\partial t^{\alpha}}=a \frac{\partial^{2} T(x, t)}{\partial x^{2}}-v \frac{\partial T(x, t)}{\partial x},
$$

where: $-\infty<x<\infty, 0<t<\infty, 0<\alpha \leq 1, a>0, v>0$. In equation (1) $\partial^{\alpha} T / \partial t^{\alpha}$ is the Caputo fractional derivative of the order $\alpha$ :

$$
\frac{\partial^{\alpha} T}{\partial t^{\alpha}}=\frac{1}{\Gamma(n-\alpha)} \int_{0}^{t}(t-\tau)^{n-\alpha-1} \frac{\partial^{n} T(\tau)}{\partial \tau^{n}} d \tau, \quad n-1<\alpha<n .
$$

Here $\Gamma(x)$ is the gamma function.

In what follows we will study the fundamental solution to the Cauchy problem for the time-fractional advection-diffusion equation (1) with the initial condition

$$
T(x, 0)=p_{0} \delta(x),
$$

where $\delta(x)$ is the Dirac delta function. In the initial condition (3) we have introduced the constant multiplier $p_{0}$ to obtain the nondimensional quantity displayed in figures.

The zero condition at infinity is also assumed:

$$
\lim _{x \rightarrow \pm \infty} T(x, t)=0 .
$$

\section{Method of solution}

To solve the Cauchy problem under consideration we use the Laplace transform with respect to time $t$. For the function $T(t), 0<t<\infty$, this transform is defined as

$$
L\{T(t)\}=T^{*}(s)=\int_{0}^{\infty} e^{-s t} T(t) d t
$$

with the inverse carrying out according to the Fourier-Mellin formula

$$
L^{-1}\left\{T^{*}(s)\right\}=T(t)=\frac{1}{2 \pi i} \int_{c-i \infty}^{c+i \infty} e^{s t} T^{*}(s) d s,
$$

where $c$ is a fixed positive number. 
For the Laplace transform rule the Caputo fractional derivative requires the knowledge of the initial values of the function and its integer derivatives of the order $k=1,2, \ldots, n-1$ :

$$
L\left\{\frac{d^{\alpha} T(t)}{d t^{\alpha}}\right\}=s^{\alpha} T^{*}(s)-\sum_{k=0}^{n-1} s^{\alpha-1-k} T^{(k)}\left(0^{+}\right), \quad n-1<\alpha \leq n .
$$

Applying the Laplace transform to equation (1) while taking into account the initial condition (3) and the rule (7) gives

$$
s^{\alpha} T^{*}(x, s)-s^{\alpha-1} p_{0} \delta(x)=a \frac{\partial^{2} T^{*}(x, s)}{\partial x^{2}}-v \frac{\partial T(x, s)}{\partial x} .
$$

Next, we use the exponential Fourier transform with respect to the spatial coordinate $x$ :

$$
\begin{aligned}
& F\{T(x)\}=\widetilde{T}(\xi)=\frac{1}{\sqrt{2 \pi}} \int_{-\infty}^{\infty} e^{i x \xi} T(x) d x, \\
& F^{-1}\{\tilde{T}(\xi)\}=T(x)=\frac{1}{\sqrt{2 \pi}} \int_{-\infty}^{\infty} e^{-i x \xi} \widetilde{T}(\xi) d \xi
\end{aligned}
$$

The Fourier transform of the derivative of a function is defined by the following relation:

$$
F\left\{\frac{d^{n} T(x)}{d t^{n}}\right\}=(-i \xi)^{n} \widetilde{T}(\xi)
$$

Application of the exponential Fourier transform (9) to equation (8) using (11) leads to

$$
s^{\alpha} \widetilde{T}^{*}(\xi, s)-s^{\alpha-1} \frac{p_{0}}{\sqrt{2 \pi}}=-a \xi^{2} \widetilde{T}^{*}(\xi, s)+i v \xi \widetilde{T}^{*}(\xi, s)
$$

and in the transform domain we get

$$
\widetilde{T}^{*}(\xi, s)=\frac{p_{0}}{\sqrt{2 \pi}} \frac{s^{\alpha-1}}{s^{\alpha}+a \xi^{2}-i v \xi} .
$$

Inversion of the Laplace and Fourier transforms results in the solution:

$$
T(x, t)=\frac{p_{0}}{2 \pi} \int_{-\infty}^{\infty} E_{\alpha}\left[-\left(a \xi^{2}-i v \xi\right) t^{\alpha}\right] e^{-i x \xi} d \xi .
$$


To obtain (14) the following formula [1]

$$
L^{-1}\left\{\frac{s^{\alpha-1}}{s^{\alpha}+b}\right\}=E_{\alpha}\left(-b t^{\alpha}\right)
$$

has been used, where $E_{\alpha}(z)$ is the Mittag-Leffler function in one parameter $\alpha$ having the series representation

$$
E_{\alpha}(z)=\sum_{n=0}^{\infty} \frac{z^{n}}{\Gamma(\alpha n+1)}, \quad \alpha>0
$$

For negative real values of the argument the Mittag-Leffler function $E_{\alpha}(-x)$ can be calculated as the integral [15]:

$$
E_{\alpha}(-x)=\frac{\sin (\alpha \pi)}{\pi} \int_{0}^{\infty} e^{-x^{1 / \alpha} u} \frac{u^{\alpha-1}}{u^{2 \alpha}+2 u^{\alpha} \cos (\alpha \pi)+1} d u, \quad 0<\alpha<1 .
$$

Next, we recast the argument of the Mittag-Leffler function in the solution (14) as

$$
T(x, t)=\frac{p_{0}}{2 \pi} \int_{-\infty}^{\infty} E_{\alpha}\left\{-a\left[\left(\xi-i \frac{v}{2 a}\right)^{2}+\frac{v^{2}}{4 a^{2}}\right] t^{\alpha}\right\} e^{-i x \xi} d \xi
$$

and use the substitution

$$
\eta=\xi-i \frac{v}{2 a}
$$

which allows us to rewrite the solution (18) in the form

$$
T(x, t)=\frac{p_{0}}{2 \pi} \int_{-\infty}^{\infty} E_{\alpha}\left[-a\left(\eta^{2}+\frac{v^{2}}{4 a^{2}}\right) t^{\alpha}\right] \exp \left[-i x\left(\eta+i \frac{v}{2 a}\right)\right] d \eta
$$

and consequently as

$$
T(x, t)=\frac{p_{0}}{2 \pi} \exp \left(\frac{v x}{2 a}\right) \int_{-\infty}^{\infty} E_{\alpha}\left[-a\left(\eta^{2}+\frac{v^{2}}{4 a^{2}}\right) t^{\alpha}\right] \cos (x \eta) d \eta .
$$

In the particular case $\alpha=1$ the Mittag-Leffler function $E_{1}(z)=e^{z}$, hence for the classical advection-diffusion equation we have 


$$
T(x, t)=\frac{p_{0}}{2 \pi} \exp \left(\frac{v x}{2 a}\right) \int_{-\infty}^{\infty} \exp \left[-a\left(\eta^{2}+\frac{v^{2}}{4 a^{2}}\right) t\right] \cos (x \eta) d \eta
$$

or

$$
T(x, t)=\frac{p_{0}}{2 \pi} \exp \left(\frac{v x}{2 a}\right) \exp \left(-\frac{v^{2} t}{4 a}\right) \int_{-\infty}^{\infty} e^{-a t \eta^{2}} \cos (x \eta) d \eta
$$

Since $[16]$

$$
\int_{-\infty}^{\infty} e^{-a t \eta^{2}} \cos (x \eta) d \eta=\frac{\sqrt{\pi}}{\sqrt{a t}} \exp \left(-\frac{x^{2}}{4 a t}\right)
$$

then

$$
T(x, t)=\frac{p_{0}}{2 \pi} \exp \left(\frac{v x}{2 a}\right) \exp \left(-\frac{v^{2} t}{4 a}\right) \frac{\sqrt{\pi}}{\sqrt{a t}} \exp \left(-\frac{x^{2}}{4 a t}\right),
$$

and we get the known fundamental solution of the classical advection-diffusion equation

$$
T(x, t)=\frac{p_{0}}{2 \sqrt{\pi a t}} \exp \left[-\frac{(x-v t)^{2}}{4 a t}\right]
$$

\section{Numerical results}

In numerical calculations we use the following nondimensional quantities:

$$
\bar{x}=\frac{x}{\sqrt{a} t^{\alpha / 2}}, \quad \bar{\eta}=\sqrt{a} t^{\alpha / 2} \eta, \quad \bar{v}=\frac{v t^{\alpha / 2}}{\sqrt{a}}, \quad \bar{T}=\frac{\sqrt{a} t^{\alpha / 2} T}{p_{0}} .
$$

Hence, the nondimensional solution takes the form

$$
\bar{T}(\bar{x})=\frac{1}{\pi} \exp \left(\frac{\bar{v} \bar{x}}{2}\right) \int_{0}^{\infty} E_{\alpha}\left(-\bar{\eta}^{2}-\frac{\bar{v}^{2}}{4}\right) \cos (\bar{x} \bar{\eta}) d \bar{\eta} .
$$

The results of numerical calculations for different values of the order $\alpha$ of the fractional derivative and of the drift parameter $\bar{v}$ are shown in Figures 1-4. 


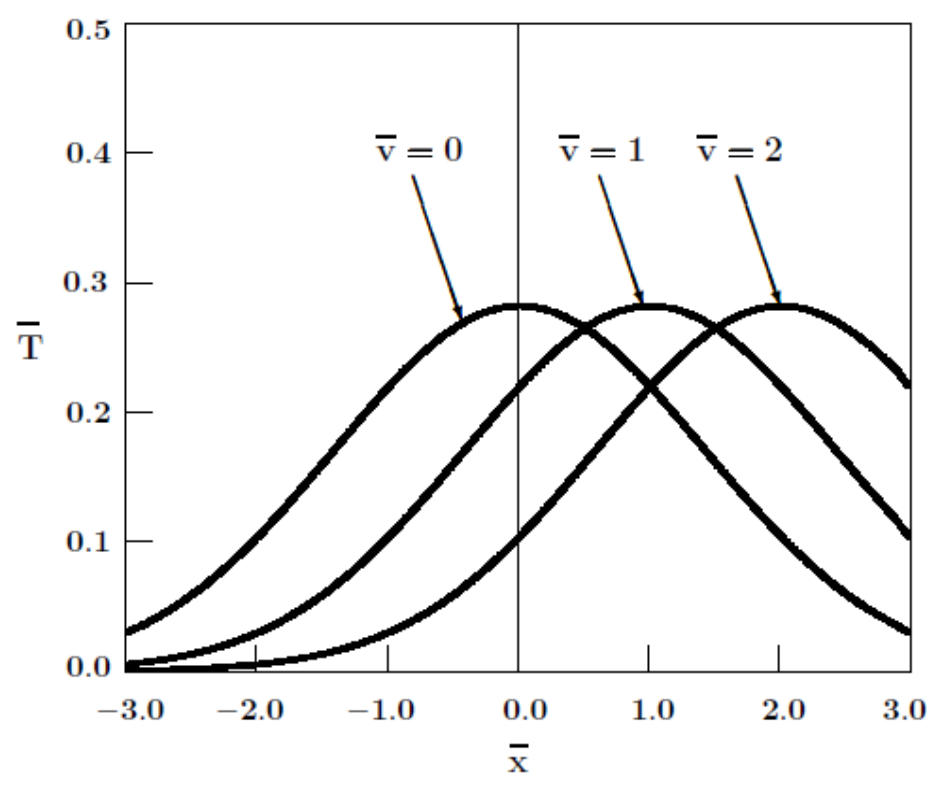

Fig. 1. Dependence of the fundamental solution to the Cauchy problem on distance; $\alpha=1$

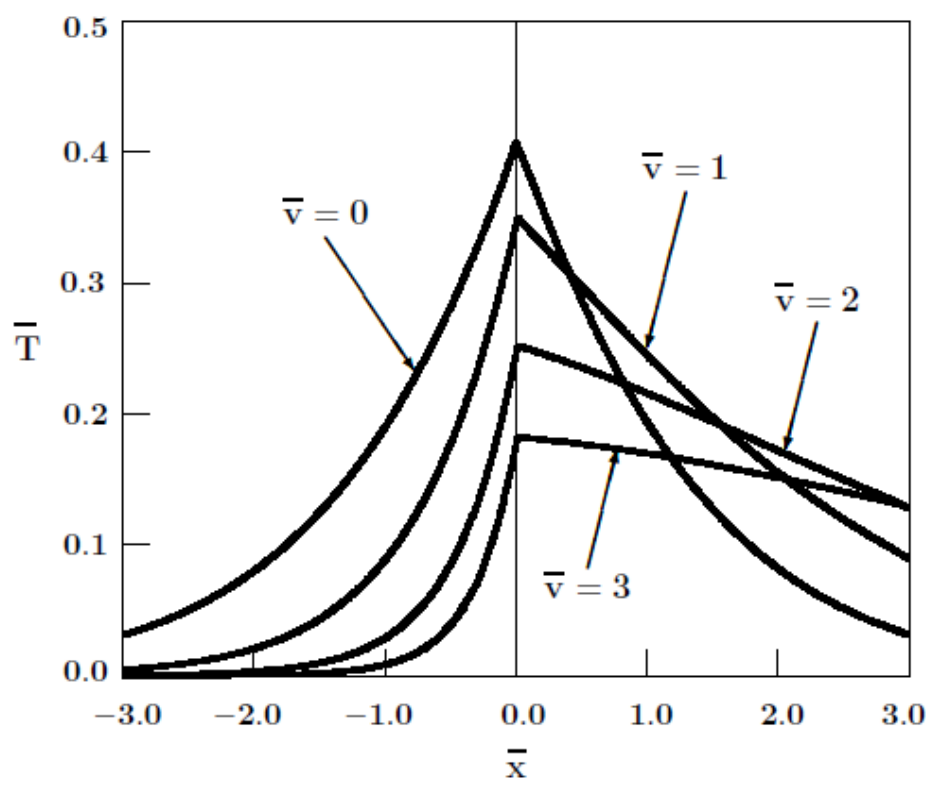

Fig. 2. Dependence of the fundamental solution to the Cauchy problem on distance; $\alpha=0.5$ 


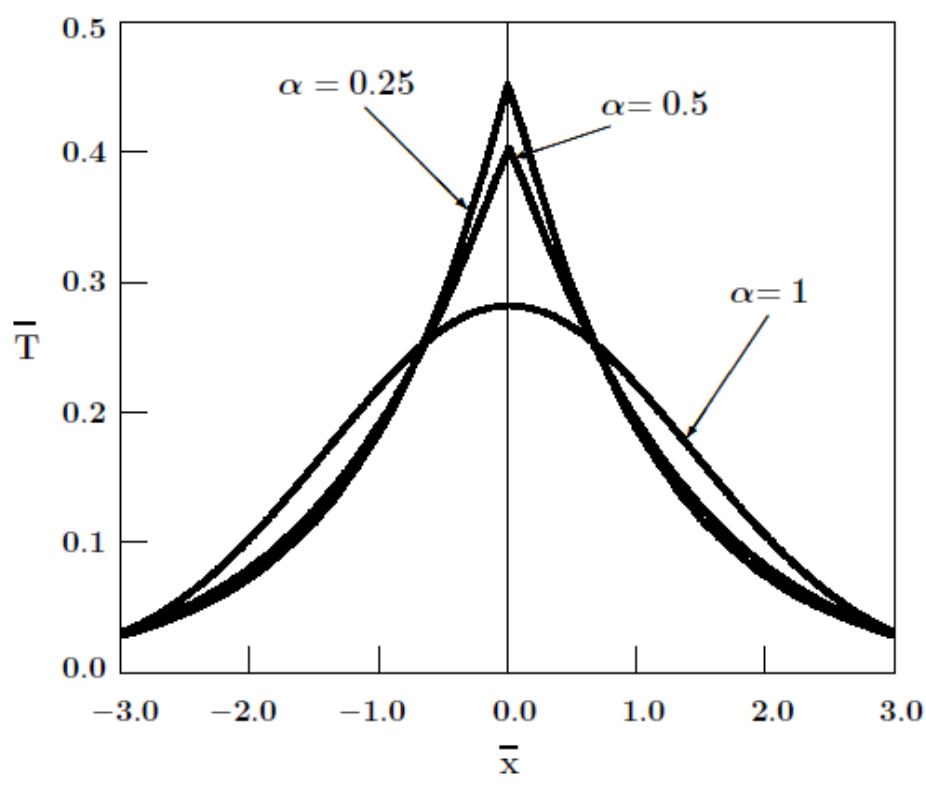

Fig. 3. Dependence of the fundamental solution to the Cauchy problem on distance; $\bar{v}=0$

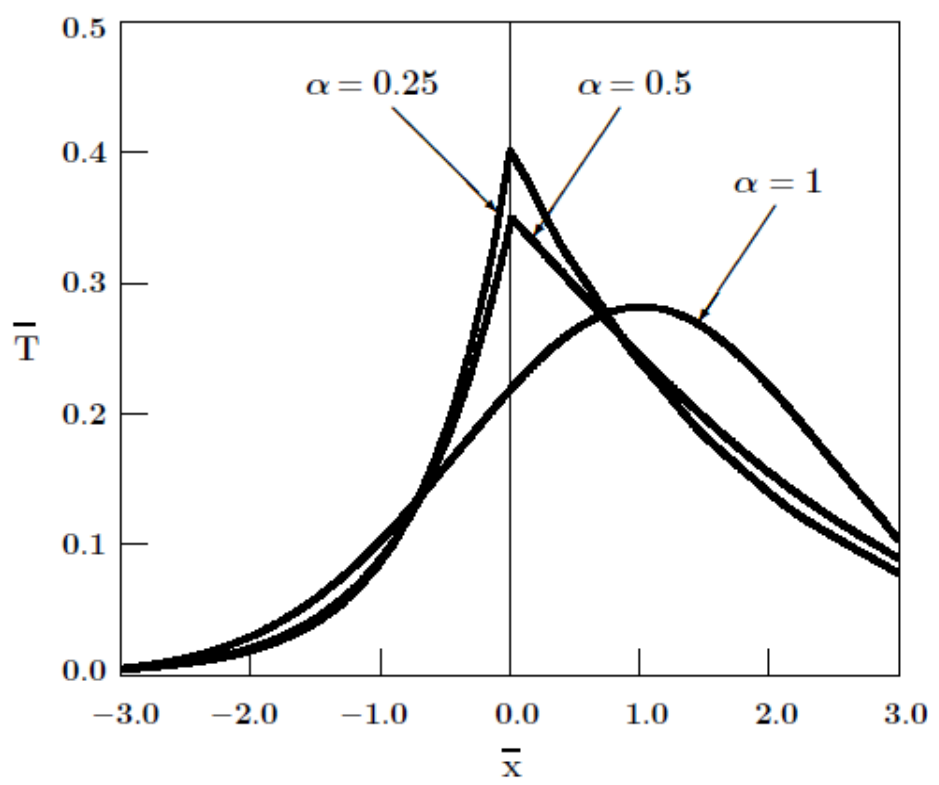

Fig. 4. Dependence of the fundamental solution to the Cauchy problem on distance; $\bar{v}=1$ 


\section{Conclusions}

We have considered the time-fractional advection-diffusion equation with the Caputo fractional derivative in the case of one spatial coordinate. The fundamental solution to the Cauchy problem has been obtained using the integral transform technique. The numerical results show the significant influence of the order of fractional derivative on the solution. In the case of the standard advection-diffusion equation $(\alpha=1)$ the quantity $v$ only causes a drift of the maximum value of the solution in the $x$-direction ( $x-v t$ in the solution (26), see Fig. 1). In the case of time-fractional advection-diffusion equation with $0<\alpha<1$ drift leads to the decreasing of the maximum value of the fundamental solution (see Fig. 2).

\section{References}

[1] Podlubny I., Fractional Differential Equations, Academic Press, San Diego 1999.

[2] Metzler R., Klafter J., The random walk's guide to anomalous diffusion: a fractional dynamics approach, Phys. Rep. 2000, 339, 1-77.

[3] West B.J., Bologna M., Grigolini P., Physics of Fractals Operators, Springer, New York 2003.

[4] Povstenko Y.Z., Fractional heat conduction equation and associated thermal stresses, J. Thermal Stresses 2005, 28, 83-102.

[5] Magin R.L., Fractional Calculus in Bioengineering, Begell House Publishers, Connecticut 2006.

[6] Klimek M., On Solutions of Linear Fractional Differential Equations of a Variational Type, The Publishing Office of Częstochowa University of Technology, Częstochowa 2009.

[7] Leszczyński J.S. An Introduction to Fractional Mechanics, The Publishing Office of Częstochowa University of Technology, Częstochowa 2011.

[8] Uchaikin V.V., Fractional Derivatives for Physicists and Engineers, Springer, Berlin 2013.

[9] Meerschaert M., Tadjeran C., Finite difference approximations for fractional advection-dispersion flow equations, J. Comput. Appl. Math. 2004, 172, 65-77.

[10] Liu F., Zhuang P., Anh V., Turner I., Burrage K., Stability and convergence of the difference methods for the space-time fractional advection-diffusion equation, Appl. Math. Comput. 2007, 191, 12-20.

[11] Merdan M., Analytical approximate solutions of fractional convection-diffusion equation with modified Riemann-Liouville derivative by means of fractional variational iteration methods, Iranian J. Sci. Techn. 2013, A1, 83-92.

[12] Liu F., Zhuang P., Turner I., Burrage K., Anh V., A new fractional finite volume method for solving the fractional diffusion equation, Appl. Math. Modell. 2013, http://dx.doi.org/10.1016 /j.apm.2013.10.007

[13] Liu F., Anh V., Turner I., Zhuang P., Time-fractional advection-dispersion equation, J. Appl. Math. Comput. 2003, 13, 233-245.

[14] Huang F., Liu F., The time fractional diffusion equation and advection-dispersion equation, ANZIAM J. 2005, 46, 317-330.

[15] Gorenflo R., Loutchko J., Luchko Yu., Computation of the Mittag-Leffler function and its derivatives, Fract. Calc. Appl. Anal. 2002, 5, 491-518.

[16] Prudnikov A.P., Brychkov Yu.A., Marichev O.I., Integrals and Series. Elementary Functions, Nauka, Moscow 1981 (in Russian). 